\title{
THE RR LYRAE DISTANCE SCALE
}

\author{
PIOTR POPOWSKI AND ANDREW GOULD \\ Department of Astronomy \\ The Ohio State University \\ 174 W. 18th Avenue, Columbus, $\mathrm{OH}$ 43210, USA.
}

\begin{abstract}
We review seven methods of measuring the absolute magnitude $M_{V}$ of RR Lyrae stars in light of the Hipparcos mission and other recent developments. We focus on identifying possible systematic errors and rank the methods by relative immunity to such errors. For the three most robust methods, statistical parallax, trigonometric parallax, and cluster kinematics, we find $M_{V}$ (at $\left.[\mathrm{Fe} / \mathrm{H}]=-1.6\right)$ of $0.77 \pm 0.13,0.71 \pm 0.15,0.67 \pm 0.10$. These methods cluster consistently around $0.71 \pm 0.07$. We find that BaadeWesselink and theoretical models both yield a broad range of possible values (0.45-0.70 and 0.45-0.65) due to systematic uncertainties in the temperature scale and input physics. Main-sequence fitting gives a much brighter $M_{V}=0.45 \pm 0.04$ but this may be due to a difference in the metallicity scales of the cluster giants and the calibrating subdwarfs. White-dwarf cooling-sequence fitting gives $0.67 \pm 0.13$ and is potentially very robust, but at present is too new to be fully tested for systematics. If the three most robust methods are combined with Walker's mean measurement for 6 LMC clusters, $V_{0, \mathrm{LMC}}=18.98 \pm 0.03$ at $[\mathrm{Fe} / \mathrm{H}]=-1.9$, then $\mu_{\mathrm{LMC}}=18.33 \pm 0.08$.
\end{abstract}

\section{Introduction}

RR Lyrae stars are among the most popular local distance indicators. Smith (1995) describes in detail their general properties. One can measure the apparent magnitudes of RR Lyrae stars in a stellar system and infer their mean deredenned apparent magnitude $V_{0}$. If the mean absolute magnitude of RR Lyrae stars $M_{V}(R R)$ at the system metallicity [Fe/H] is known, then the system's distance modulus $\mu$ is:

$$
\mu=V_{0}-M_{V}(R R)
$$


In the following sections, we will assume that $V_{0}$ can be measured accurately and concentrate on the $M_{V}$ determinations. $M_{V}$ can be calibrated through field or globular cluster stars. There are compelling arguments (e.g., Catelan 1998) against two distinct, environment-dependent $M_{V}$ scales (i.e., distance scales), and we will seek a universal absolute magnitude - metallicity $M_{V}$ $-[\mathrm{Fe} / \mathrm{H}]$ relation:

$$
M_{V}(R R)=\alpha([\mathrm{Fe} / \mathrm{H}]+1.6)+\beta .
$$

Most of the extragalactic distance scale is tied to the Large Magellanic Cloud (LMC). The $M_{V}-[\mathrm{Fe} / \mathrm{H}]$ relation from (2) guarantees that $\mu_{L M C}$ will be sensitive to the zero point $\beta$ and insensitive to the slope $\alpha$. Therefore, we do not discuss many $\alpha$ determinations (Ajhar et al. 1996; Fusi Pecci et al. 1996; Kovács \& Jurcsik 1996 etc.), but concentrate on methods yielding $\beta$. Note that $V_{0, \mathrm{LMC}} \approx 19.0$ (Walker 1992 ; Hazen \& Nemec 1992 ; Reid \& Freedman 1994) and so, from equation (1) the division between the short and long distance scale occurs at $\beta \sim 0.55$, with fainter $M_{V}$ corresponding to the short distance scale. Some methods determine $M_{V}(R R)$ directly using their positions (trigonometric parallax), kinematics (statistical parallax) or pulsational properties (Baade-Wesselink). Others (cluster kinematics, main sequence and white dwarf fitting) establish globular cluster distances and then, in a second step, $M_{V}(R R)$. We group methods according to their mathematical description. Our, rather incomplete, literature review serves mostly illustrative purposes, as we concentrate on the physical picture of the methods, emphasizing their strengths and weaknesses.

\section{Kinematic Methods}

The distance to an ensemble of stars can be measured by comparing their radial velocities $\left(\mathrm{km} \mathrm{s}^{-1}\right)$ and proper motions $\left(\mathrm{s}^{-1}\right)$ under the assumption that these are due to (statistically) identical physical velocities. In effect, one fixes the distance so that either the first moment of the population (bulk motion) as determined from the proper motions is equal to first moment as determined from the radial velocities, or so that the second moments (dispersions) are equal, or both.

The great beauty of these methods is that the basic measurements are of dimensionless quantities (redshift for radial velocities and positions on the sky for proper motions) and therefore no assumptions about the distance scale enter the determinations. The major systematic uncertainty (aside from concerns about the quality of the data) is that it may be difficult to verify that the radial velocities and proper motions in fact arise from the same physical velocity distribution.

One may show that if the measurement errors are small compared to the intrinsic dispersion of the population, then the fractional distance error 
from equating the first moments is $\delta \eta / \eta=1 /\left[\left(A_{1} N\right)^{1 / 2} \kappa \sin \theta\right]$ where $N$ is the total number of stars, $\theta$ is the angular size of the system, $A_{1}$ is a geometrical factor of order unity, and $\kappa$ is the ratio of bulk motion to velocity dispersion. Similarly the error from equating the second moments is $\delta \eta / \eta=1 /\left(A_{2} N\right)^{1 / 2}$ where $A_{2}$ is another factor of order unity. Hence, by combining the two methods, the error is

$$
\left(\frac{\delta \eta}{\eta}\right)^{2}=\frac{1}{N\left[A_{1}(\kappa \sin \theta)^{2}+A_{2}\right]}
$$

For nearby open clusters like the Hyades, $\kappa \sim 10^{2}$ and $\theta \sim 10^{-1}$, so the first term in the denominator dominates, and the distance is determined from the first moments. This is sometimes called the "moving cluster" method. However, there are no RR Lyrae stars in open clusters. For globular clusters, $\kappa \sim 10^{1}$ and $\theta \sim 10^{-3}$, so the second term dominates. Hence, kinematic distances to globular clusters are determined by matching velocity dispersions. For field RR Lyrae stars in the solar neighborhood, $\kappa \sim 2.1$ and $\sin \theta \sim 1$, so both the first and second moments are used to determine the RR Lyrae distance scale. This is called "statistical parallax". Statistical parallax and kinematic distance to globular clusters have different sources of systematic error, so we treat them separately.

\subsection{STATISTICAL PARALLAX}

Statistical parallax is reviewed thoroughly by Layden elsewhere in this volume, so we give only a brief overview here. In contrast to the case of globular clusters, local field RR Lyrae stars are not at a common distance. Hence, before their radial velocities and proper motions can be compared, the stars must be put on a common scale by taking account of their dereddened apparent magnitudes. That is, one can measure the 9 parameters describing the velocity ellipsoid (3 components of bulk motion plus 6 independent components of the velocity-dispersion tensor) from radial velocities alone. On the other hand, if one assumes some arbitrary $M_{V}(R R)$, for the RR Lyrae stars, then one can infer their distances from their measured apparent magnitudes and estimated extinctions. The distances and proper motions yield the transverse velocities, and from these one can again estimate the 9 parameters of the velocity ellipsoid. One could then adjust the assumed $M_{V}$ so that the velocity ellipsoid from proper motions matched the velocity ellipsoid from radial velocities as closely as possible. In practice, one fits for all 10 parameters (9 for the velocities plus $M_{V}$ ) simultaneously using maximum likelihood. The maximum likelihood approach was pioneered by Clube \& Dawe (1980) and was subsequently applied by Hawley et al. (1986) to the then best-available data 
set of 142 RRab stars. They obtained $M_{V}=0.76 \pm 0.14$. Note that the error is very close to the theoretical minimum (for no measurement errors) $\sigma_{M_{V}}=(5 / \ln 10)\left[(2 N / 9)\left(6+\kappa^{2}\right)\right]^{-1 / 2}=0.12 \mathrm{mag}$ (Popowski \& Gould 1998a). This is because the measurement errors do not contribute significantly if they are substantially below the velocity dispersion.

As we discuss below, this result is fainter than virtually all other estimates and much fainter than some. Since the method itself appears extremely robust, several workers have invested substantial effort to obtain the most reliable input data and to investigate whether any unrecognized effects could be leading to systematic errors. Layden (1994) and Layden et al. (1996) put all existing data on a homogeneous system. They incorporated the proper motions from the new Lick NPM1 (Klemola, Hanson, \& Jones 1993) survey which has smaller (and just as important, better-understood) errors than previous proper-motion studies. They used the Lick catalog to calibrate the errors of the non-Lick proper motions. They found that the diverse photometry sources were offset from one another and put these on a common zero point. Likewise, they put all the extinctions on the Burstein \& Heiles (1984) system. They found $M_{V}=0.71 \pm 0.12$ for 162 "halo-3" RRab stars with mean metallicity $\langle[\mathrm{Fe} / \mathrm{H}]\rangle=-1.61$. Popowski \& Gould (1998a) developed a formulation of maximum likelihood which permitted both new analytic investigations and much more vigorous Monte Carlo investigations of possible systematic effects. These included a possible change in the velocity ellipsoid with distance from the Galactic plane, rotation of the local coordinate frames relative to the Sun's frame, and effects due to the severe non-Gaussianity of the velocity distribution. However, these effects all proved negligible. The most important previously overlooked effect that they found was Malmquist bias, and they obtained $M_{V}=0.76 \pm 0.12$ for the 162-star Layden et al. (1996) sample.

Hipparcos has had two major impacts on the RR Lyrae statistical parallax determination. One is, of course, new and more precise proper-motion measurements. The other, more surprisingly and indeed more importantly, is better photometry. Fernley et al. (1998a) fit Hipparcos light curves to obtain new photometry for most RR Lyrae stars in the Hipparcos catalog. They combined these with Hipparcos proper motions and obtained $M_{V}=0.77 \pm 0.17$ for 84 "halo" $([\mathrm{Fe} / \mathrm{H}]<-1.3)$ stars with $\langle[\mathrm{Fe} / \mathrm{H}]\rangle=-1.66$, including 69 RRab's and 15 RRc's. Fernley et al. (1998a) also used highprecision ground-based photometry to show that their Hipparcos-based mean magnitudes were correct with very small $(<0.02 \mathrm{mag})$ scatter. (Tsujimoto, Miyamoto, \& Yoshii 1998 conducted a similar study of 99 Hipparcos "halo" stars and found $M_{V}=0.69 \pm 0.10$ at $\langle[\mathrm{Fe} / \mathrm{H}]\rangle=-1.58$. However, since their quoted error is a factor 0.7 below the theoretical minimum, we conclude that their analysis is incorrect.) Popowski \& Gould (1998b) used 
the Hipparcos proper motions to check earlier catalogs and found that only the Lick catalog is of sufficiently high quality to use. Gould \& Popowski (1998) used Fernley et al.'s (1998a) Hipparcos-based mean magnitudes to check Layden et al.'s (1996) systematization of previous heterogeneous photometry, and found that it was $0.06 \mathrm{mag}$ too bright. They also incorporated the new extinction map of Schlegel, Finkbeiner \& Davis (1998) based on COBE/IRAS measurements of dust emission, and they eliminated a number of stars with questionable extinctions and proper motions to obtain $M_{V}=0.77 \pm 0.13$ for 147 "halo-3" RRab stars with $\langle[\mathrm{Fe} / \mathrm{H}]\rangle=-1.60$.

Layden et al. (1996) noted that the velocity ellipsoid of their solution is in good agreement with that of Beers \& Sommer-Larsen (1995) for metalpoor stars and took this as independent confirmation of the correctness of their results. Popowski \& Gould (1998b) and Gould \& Popowski (1998) directly incorporated the Beers \& Sommer-Larsen stars into the analysis and obtained $M_{V}=0.80 \pm 0.11$ at $\langle[\mathrm{Fe} / \mathrm{H}]\rangle=-1.71$ for a combined sample based on 149 RRab Lyrae stars and 716 non-RR Lyrae stars.

At this point, essentially all systematic errors have been removed from the RR Lyrae statistical parallax determination. The statistical errors in the above two solutions $\left(M_{V}=0.77 \pm 0.13\right.$ at $\langle[\mathrm{Fe} / \mathrm{H}]\rangle=-1.60$ or $M_{V}=$ $0.80 \pm 0.11$ at $\langle[\mathrm{Fe} / \mathrm{H}]\rangle=-1.71)$ should therefore be taken at face value.

\subsection{KINEMATIC CLUSTER DISTANCES}

If the distance to a cluster is known, the $M_{V}(R R)$ can be determined by subtracting the distance modulus from the dereddened apparent magnitude of RR Lyrae stars in the cluster, or more generally from the height of the zero age horizontal branch (ZAHB) at the color of the instability strip. Cluster distances can be determined kinematically by comparing the dispersions of the radial velocities and proper motions. The principle is similar to statistical parallax, but there are two major practical differences. First, the proper motions are much smaller for clusters $\left(\sim 10 \mathrm{~km} \mathrm{~s}^{-1} / 10 \mathrm{kpc}=0.2 \operatorname{mas~yr}^{-1}\right)$ than for nearby field RR Lyrae stars $\left(\sim 200 \mathrm{~km} \mathrm{~s}^{-1} / 2 \mathrm{kpc}=20\right.$ mas yr$\left.^{-1}\right)$. Second, clusters are seen in projection, so the 3 -space position of individual stars is unknown. This introduces additional systematic effects that are difficult to fully take into account.

Cudworth (1979) made the first such measurement, finding a distance $d=9.6 \pm 2.6 \mathrm{kpc}$ for M3 by comparing the proper-motion dispersion of

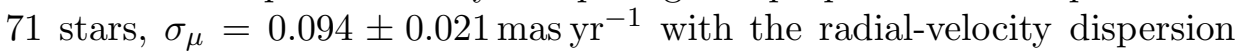
from Gunn \& Griffin (1979), $\sigma_{r}=4.3 \pm 0.7 \mathrm{~km} \mathrm{~s}^{-1}$. The measured rms dis-

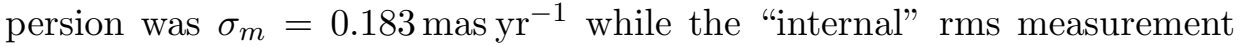

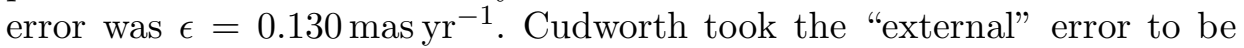
$f \epsilon$ and estimated $f=1.20 \pm 0.12$. Note that the measurement errors were 
actually larger than the cluster dispersion $\sigma_{\mu}^{2}=\sigma_{m}^{2}-(f \epsilon)^{2}$ and that the correction factor $f$ is therefore very important. This factor had earlier been measured as $f=1.1 \pm 0.1$ by Cudworth \& Monet (1979) by comparing reductions of two different plate sets of M13, one weak and one deep. However, Cudworth (1979) argued that this was only a lower limit on $f$ since the two sets were taken with the same Yerkes telescope, over the same time interval, ca. 1900 to ca. 1975 . We note that a perfectly plausible $f \equiv 1$ would yield $\sigma_{\mu}=0.129 \pm 0.023$ mas yr$^{-1}$ and hence a cluster distance of $d=7.1 \pm 1.7 \mathrm{kpc}$.

Cudworth (1979) assumed that M3 is isotropic because it looks circularly symmetric on the sky and rotation is barely detectable if at all in the radial velocities. Hence, he simply divided the radial-velocity dispersion by the proper-motion to obtain the distance. However, if the cluster rotation axis were along the line of sight, these observational characteristics would remain in tact, but the radial dispersion would no longer be representative of the tangential dispersion. (Transverse rotation cannot be measured from the proper-motion data because there are too few foreground stars and their dispersion is too large to form a stable framework.) Thus, M3 exemplifies both the promise and the problems of this technique.

Gunn \& Griffin (1979) introduced a major advance by incorporating Mitchie-King models (Mitchie 1963) into the analysis, and Lupton, Gunn, \& Griffin (1987) built on their experience when they measured the distance to M13. The Mitchie-King models are constrained by the light profile, the radial-velocity map, and the mass function. Such models can take account of rotation although the degeneracy noted in the previous paragraph remains. M13 is closer than M3 (6.5 vs. $9.6 \mathrm{kpc}$ ), the velocity dispersion is larger (6.5 vs. $4.3 \mathrm{~km} \mathrm{~s}^{-1}$ ), and the number of stars is larger (268 vs. 71 ). Hence the distance errors are much smaller ( $8 \%$ vs. $27 \%$ ). Lupton et al. (1987) do not say whether they incorporated Cudworth \& Monet's (1979) estimate of $f=1.1$, or whether they used $f=1$. In this case, the difference in the final result is $4 \%$, or $0.08 \mathrm{mag}$ in the height of the horizontal branch (HB). Lupton et al. (1987) determined the distance only for their best-fit model of the cluster and did not obtain distances for a range of acceptable models which would have allowed them to evaluate the systematic uncertainty due to their modeling procedure.

Peterson \& Cudworth (1994) measured the distance to M22 by equating the minor axis proper-motion dispersion with the radial-velocity dispersion and found $d=2.6 \pm 0.3 \mathrm{kpc}$. That is, they assumed that the axis of the observed rotation is in the plane of the sky and, rather than attempt to model the effect of this rotation on the observed proper motions in the symmetry plane, simply ignored half the proper-motion data. They do not say whether they incorporated an $f$ factor, but for this case the difference 
between $f=1$ and $f=1.1$ is only 0.03 mag.

Rees (1996) has applied Gunn \& Griffin's (1979) Mitchie-King technique to 8 clusters (47 Tuc, M5, M4, M92, N6397, M22, M15, and M2) and combined the results with the distances obtained for M3 and M13 by Cudworth (1979) and Lupton, Gunn \& Griffin (1987). Subsequently, Rees (1998 priv. comm.) rereduced the M22 data and derived a distance, $d=11.26 \pm 1.31 \mathrm{kpc}$. He also came to the conclusion that the N6397 proper motions were not reliable enough to use. Two of the remaining 9 clusters have features which are not well represented by Mitchie-King models: M15 has a central cusp, and 47 Tuc has differential rotation. If these two clusters are eliminated, the remaining seven have a mean zero-age HB (ZAHB) of $M_{V}=0.62 \pm 0.10 \mathrm{mag}$ at $\langle[\mathrm{Fe} / \mathrm{H}]\rangle=-1.6$. (Rees quotes an uncertainty of $0.05 \mathrm{mag}$, evidently having renormalized the errors because $\chi^{2}=1.66$ for 6 degrees of freedom. However, since the errors are basically due to counting statistics - and so cannot have been overestimated - the errors should not be renormalized.) If M15 and 47 Tuc are re-included, $M_{V}=0.58 \pm 0.08$.

The details of Rees's (1996) work are not yet publicly available, so it is impossible to give a complete assessment of the systematic errors. Clearly, if the proper motion errors have been systematically underestimated (as suspected by Cudworth \& Monet 1979 and Cudworth 1979), then the luminosity of the ZAHB has been underestimated for each of the clusters. This is a much smaller effect for nearer clusters which have smaller overall errors and so more statistical weight. Alternatively, if there is no error underestimate but an underestimate has been corrected for, then the luminosity of the ZAHB has been overestimated. To date, no one has published a set of distances for a set of acceptable Mitchie-King models, so there is no way to assess the systematic error due to uncertainty in the cluster geometry. At least until such tests are conducted, clusters that are inconsistent with Mitchie-King models (i.e. M15 and 47 Tuc) should be excluded.

Finally, there is bias introduced by binaries which increase the radialvelocity dispersion but not the proper-motion dispersion (measured on 100 yr time scales). The correction for this previously unrecognized bias makes $M_{V}$ fainter by $\delta M_{V}=(2.5 / \ln 10) f\left\langle\left(\gamma V_{\text {orb }}\right)^{2}\right\rangle / 3 \sigma^{2}$ mag, where $f$ is the binary fraction, $\sigma$ is the 1-dimensional dispersion of the cluster, $V_{\text {orb }}$ is the mean orbital velocity of the binaries, $\gamma=M_{s} /\left(M_{s}+M_{p}\right)$, and $M_{p}$ and $M_{s}$ are the primary and secondary masses. Hut et al. (1992) conclude (primarily based on Pryor et al. 1989) that for periods of 0.2 to 20 years, the binary fraction of globular clusters is consistent with that of $\mathrm{G}$ stars as measured by Duquennoy \& Mayor (1991), i.e., 8\%. Applying the above formula to the Duquennoy \& Mayor (1991) distribution, we find $\delta M_{V}=0.05$. Hence, our best estimate for this method is $M_{V}=0.67 \pm 0.10$ at $\langle[\mathrm{Fe} / \mathrm{H}]\rangle=-1.6$. We caution that this error is only statistical and that the systematics are 
not fully understood.

\section{Trigonometric Parallax}

Prior to Hipparcos, it was not possible to make a useful estimate of the $M_{V}(R R)$ using trigonometric parallax. Hipparcos measured only one RR Lyrae star with reasonable precision, RR Lyrae itself. This yields $M_{V}=$ $0.78 \pm 0.29$ at $[\mathrm{Fe} / \mathrm{H}]=-1.39$ (Fernley et al. 1998a), still not precise enough to discriminate among various other estimates.

However, Gratton (1998) has extended this approach by considering all HB stars (not just RR Lyrae) in a magnitude limited sample, $V_{0}<9$. This criterion yields 22 stars, including 10 blue HB stars, 3 RR Lyrae stars, and 9 red HB stars. Gratton (1998) obtains $M_{V}=0.69 \pm 0.10$ at $\langle\mathrm{Fe} / \mathrm{H}\rangle=-1.41$ if all 22 stars are included, or $M_{V}=0.60 \pm 0.12$ at $\langle\mathrm{Fe} / \mathrm{H}\rangle=-1.51$ if one red HB star that he suspects of being a giant (not HB) star is eliminated. We first review and correct Gratton's statistical procedure, and then discuss more carefully the problem of selection.

Gratton (1998) begins by determining the shape of the HB from observations of M5. This yields $\delta M_{V}(B-V)$, the difference between the magnitude at the instability strip and the magnitude of a star at a particular $B-V$ color. His model of the HB is therefore characterized by a single parameter, $M_{V}$ at the instability strip. From the observed magnitude and color and the inferred reddening, he is then able to predict the parallax as a function of this parameter,

$$
\pi^{*}\left(M_{V}\right)=10^{0.2\left\{\left[M_{V}-\delta M_{V}(B-V)\right]-[V-3.1 E(B-V)]+10\right\}} \operatorname{mas}
$$

He then forms the average of the difference between this quantity and the observed parallax, $\pi$, weighted by the observational errors, and determines $M_{V}$ by setting this quantity to zero

$$
0 \equiv \Delta=\sum_{i} \frac{\pi_{i}^{*}\left(M_{V}\right)-\pi_{i}}{\sigma_{\pi, i}^{2}}
$$

Now, the proper procedure would be to form $\chi^{2}\left(M_{V}\right)=\sum_{i}\left(\pi_{i}^{*}\left(M_{V}\right)-\right.$ $\left.\pi_{i}\right)^{2} / \sigma_{\pi, i}^{2}$ and then to minimize it with respect $M_{V}$. Formally,

$$
0 \equiv \frac{\partial \chi^{2}\left(M_{V}\right)}{\partial M_{V}}=\frac{\ln 10}{2.5} \sum_{i} \frac{\pi_{i}^{*}\left(M_{V}\right)-\pi_{i}}{\sigma_{\pi, i}^{2}} \pi_{i}^{*}\left(M_{V}\right) .
$$

Comparing equations (5) and (6), we see that Gratton (1998) has in effect weighted the terms by their distance, which of course gives higher weight to the most poorly determined values. Employing equation (6) rather than (5), we find $M_{V}=0.75 \pm 0.09$ and $M_{V}=0.64 \pm 0.12$ for the two cases. 
The red HB star that Gratton (1998) eliminated is suspicious only because it is significantly fainter than the HB. Of course, since its parallax is only $1.3 \sigma$ larger than predicted, this could be a normal statistical fluctuation. There does not appear to be any way to guard against giant-star contamination of the red HB sample without biasing the sample by eliminating stars with above average, but normally distributed, parallaxes. Therefore the most prudent procedure is to eliminate all red HB stars. We then find $M_{V}=0.68 \pm 0.14$ at $\langle\mathrm{Fe} / \mathrm{H}\rangle=-1.62$. Finally, we note that account must be taken of the intrinsic scatter of the population. For homogeneous populations (e.g. globular clusters) the scatter in $M_{V}$ is $\sigma_{M_{V}} \sim 0.08$ mag. The upper limit for the scatter in a general field population is $\sigma_{M_{V}} \sim 0.17 \mathrm{mag}$, but a more plausible estimate is $\sigma_{M_{V}} \sim 0.14 \mathrm{mag}$ (Popowski \& Gould 1998a). We adopt that here and find $M_{V}=0.68 \pm 0.15$. This scatter introduces a Malmquist bias. One may show that if the parallax errors scale with flux as $\sigma_{\pi} \propto F^{-\nu}$, then the Malmquist bias is $\delta M_{V}=(\ln 10 / 5)(1-\nu) \sigma_{M_{V}}^{2} \sim 0.01 \mathrm{mag}$, where $\nu \sim 0.2$ (appropriate for $6.5 \leq V \leq 9$ for Hipparcos). Our final estimate is therefore $M_{V}=0.69 \pm 0.15$ at $\langle\mathrm{Fe} / \mathrm{H}\rangle=-1.62$, where the metallicity is on the Gratton scale, corresponding to $M_{V}=0.72 \pm 0.15$ at $\langle\mathrm{Fe} / \mathrm{H}\rangle=-1.6$ on the Zinn \& West (1984) scale.

\section{Baade-Wesselink Method}

The goal of the Baade-Wesselink method is to determine the equilibrium radius of a pulsating star through the combined analysis of its radial-velocity and light curves. The equilibrium radius and effective temperature, $T_{\text {eff }}$, inferred from multicolor photometry allows one to determine the luminosity, $L$. The radial-velocity curve reflects the absolute change in radius $R$ of a star, $\delta R$, due to its contraction or expansion with velocity $V_{\text {rad }}(t)$. Consequently, during the time interval $\delta t$ :

$$
\delta R(t, t+\delta t)=\bar{V}_{\text {rad }}(t, t+\delta t) * \delta t,
$$

where $\bar{V}_{\text {rad }}(t, t+\delta t)$ is the mean radial velocity in a time interval $(t, t+\delta t)$. The flux of a spherical blackbody at distance $d$ is given by:

$$
F=\frac{4 \pi \sigma_{S} R^{2} T_{\mathrm{eff}}^{4}}{d^{2}}
$$

where $\sigma_{S}$ is a constant. Consequently, one can determine $R$ by:

$$
R=2 \delta R\left(\frac{T_{\mathrm{eff}}^{4}(R) F(R+\delta R)}{T_{\mathrm{eff}}^{4}(R+\delta R) F(R)}-1\right)^{-1}
$$


In equation (9), $\delta R$ is known from radial velocity curve [eq. (7)], the fluxes are observables, and the temperature at each phase is estimated from colors based on stellar atmospheric models (e.g., Kurucz 1992).

Most of the results obtained before 1994 for field RR Lyrae stars (Liu \& Janes 1990; Jones et al. 1992; Cacciari, Clementini \& Fernley 1992; Skillen et al. 1993) can be summarized by one equation:

$$
M_{V}=0.70+0.21([\mathrm{Fe} / \mathrm{H}]+1.6)
$$

resulting in $\mu_{\mathrm{LMC}} \approx 18.3$.

The Baade-Wesselink method is more involved and model-dependent than geometric and kinematic determinations. The simple physical picture of the method presented above gives an immediate insight into its possible systematics. First, $\bar{V}_{r a d}$ in equation $(7)$ is not the mean radial velocity that is measured from spectral lines. $\bar{V}_{\text {rad }}$ would be equal to the measured velocity if the stellar photosphere were moving straight toward or away from the observer. In fact it is moving radially inward or outward from the center of a star and its direction forms some angle, $\theta$, with the observerstar line of sight, which reduces the observed radial velocity by a factor of $\cos (\theta)$. Additionally, a contribution of a given stellar surface patch to the line intensity depends on limb darkening of the star and the relative depths at which a given line and the stellar continuum form. All these effects are usually parametrized by a factor " $p$ " which is supposed to convert the measured radial velocity to a true one (e.g., Fernley 1994). Getting (1935) obtains $p=1.41$ analytically using the Milne-Eddington limb darkening model. More sophisticated investigations using model atmospheres usually produce slightly lower values in the range $1.28-1.39$. The factor $p$ is a function of pulsation phase, but Fernley et al. (1989) showed for X Ari that using a varying value of $p$ changes the derived $R$ by less than $1 \%$. The factor $p$ also increases sharply with decreasing line strength (Karp 1975). The uncertainty in $p$ does not influence the slope of the $M_{V}-[\mathrm{Fe} / \mathrm{H}]$ relation but does have a substantial impact on the zero point because $F \propto p^{2}$ [eqs. (9), (7) into (8)]. For small changes in $p$, the zero-point shift is

$$
\Delta \beta=-\frac{5}{\ln 10} \frac{\Delta p}{p}
$$

Fernley (1994) argues that the original values of RR Lyrae $p$ coefficients with a mean of $\sim 1.32$ (Skillen et al. 1993) should all be replaced with a universal value of $p=1.38$. Equation (11) then predicts $\Delta \beta=0.09$, but more detailed analysis (Fernley 1994) gives $\Delta \beta=0.07$, leading to

$$
M_{V}=0.63+0.21([\mathrm{Fe} / \mathrm{H}]+1.6) .
$$


Second, the unambiguous determination of the effective temperature from stellar colors is still problematic. Temperature differences are likely to affect both the slope and zero point of the $M_{V}-[\mathrm{Fe} / \mathrm{H}]$ relation. $T_{\text {eff }}$ as obtained from optical colors $(\lambda \lesssim 800 \mathrm{~nm})$ are higher by $200-300 \mathrm{~K}$ than those inferred from $(V-K)$ colors. Most observers prefer $(V-K)$ and usually argue that the existence of $(U-B)$ excess in some pulsational phases is an indication of atmospheric shocks. Therefore, optical colors are likely to give an incorrect $T_{\text {eff }}$. However, McNamara (1997) claims that Preston \& Paczynski (1964) find no correlation between ultraviolet excess and strength of hydrogen emission lines suggesting that some other phenomenon is responsible for this effect. He also argues that surface gravities as inferred from optical colors match much better the expectations from mass and radius values for RR Lyrae stars, whereas the surface gravities derived from $(V-K)$ are too small. McNamara (1997) derives an $M_{V}-$ $[\mathrm{Fe} / \mathrm{H}]$ relation with a rather steep slope and bright zero-point:

$$
M_{V}=0.50+0.29([\mathrm{Fe} / \mathrm{H}]+1.6) .
$$

Which set of colors should be used to obtain the $M_{V}-[\mathrm{Fe} / \mathrm{H}]$ relation remains an open question. Feast (1997) argues that even "traditional" BaadeWesselink analyses produce steep slopes. He claimes that $M_{V}$ should be treated as the independent variable rather than $[\mathrm{Fe} / \mathrm{H}]$. This is incorrect. If a regression is done, the quantity with smaller relative errors should be the independent variable. Maximum likelihood, which treats both errors equally is the most rigorous approach and reproduces the shallow slope (Fernley et al. 1998b). In addition, Feast's (1997) claim that we do not see the brightest RR Lyrae stars due to a bias of the same sort of Malmquist bias is unlikely. A more natural explanation for the lack of stars with $M_{V}<0.5$ is that they do not exist: the $M_{V}-[\mathrm{Fe} / \mathrm{H}]$ relation may simply flatten at low $[\mathrm{Fe} / \mathrm{H}]$ as predicted by some theoretical models (e.g. Caputo 1997).

Third, RR Lyrae stars are not perfect blackbodies and bolometric corrections must be applied. Even though RR Lyrae stars are rather hot and metal poor, so departures from blackbody should be modest, the adopted bolometric corrections actually differ by up to $0.1 \mathrm{mag}$ (Sandage 1993 vs. Kurucz 1992).

Similar investigations have been performed for globular cluster RR Lyrae stars. Representative results obtained by Storm, Carney \& Latham (1994), namely $M_{V}=0.66$ at $[\mathrm{Fe} / \mathrm{H}]=-1.4$ for $\mathrm{M} 5$ and $M_{V}=0.45$ at $[\mathrm{Fe} / \mathrm{H}]=$ -2.24 for M92, are slightly brighter than values predicted by equation (10). 


\section{Theoretical Models of Horizontal Branch}

One can study the $M_{V}-[\mathrm{Fe} / \mathrm{H}]$ relation using a purely theoretical approach by constructing mock HBs in the $T_{\text {eff }}-L$ plane and evolving them in time. The results depend sensitively on the input physics (especially the opacities and equation of state: Castellani, Brocato \& Persimoni 1996), He abundance, and assumed core mass. The treatment of convection is also one of the most essential ingredients. To compare theoretical HBs to HBs observed in globular clusters one must transform from the theoretical $T_{\text {eff }}-L$ plane to the $M_{V}$ - color plane. This step requires accurate atmosphere models (e.g. Kurucz 1992). Then one can investigate HB morphology, how HB appearance depends on different parameters (e.g., age) etc. One can also establish how $M_{V}(\mathrm{ZAHB})$ at the instability strip scales with the $[\mathrm{Fe} / \mathrm{H}]$ of a given stellar ensemble and how this relation depends on the evolutionary stage of the population. With some (even conservative) observational constraints taken into account, the model predictions become quite robust. Another approach involves the analysis of the pulsational properties of RR Lyrae stars in the spirit of the pioneering work by van Albada \& Baker (1971). Pulsational models predict the relations between $T_{\text {eff }},[\mathrm{Fe} / \mathrm{H}]$, luminosity, $L$, period, $P$, and mass, $M$, of the stars; e.g.,

$$
\log P=11.497+0.84 \log L-0.68 \log M-3.48 \log T_{\text {eff }}
$$

They also enable one to investigate the location of blue and red, fundamental and first overtone instability strip boundaries (Bono et al. 1995). Which regions of the instability strip are populated by RRab and RRc stars correlate with the Oosterhoff type of the cluster and gives insight into the source of the period-shift effect (Sandage 1981,1982; Bono et al. 1995) which has been used to determine the $M_{V}-[\mathrm{Fe} / \mathrm{H}]$ relation. The most robust constraints on the $M_{V}-[\mathrm{Fe} / \mathrm{H}]$ relation come from combining the pulsational and evolutionary scenarios.

The predictions of theoretical evolutionary models are sensitive to every change in input physics. For example, recent improvements in opacities and the equation of state led to an increase in HB luminosities by $\sim 0.1$ mag. Gratton et al. (1997) report an unpublished $M_{V}-[\mathrm{Fe} / \mathrm{H}]$ relation by VandenBerg et al. (1998):

$$
M_{V}(Z A H B)=0.19([\mathrm{Fe} / \mathrm{H}]+1.6)+0.66
$$

Salaris et al. (1997) obtain a slightly brighter result with a similar slope:

$$
M_{V}(Z A H B)=0.21([\mathrm{Fe} / \mathrm{H}]+1.6)+0.57
$$

However HB luminosities are very sensitive to the He core mass. Mazzittelli et al. (1995) suggested that core masses should be increased by $0.01 M_{\odot}$ 
relative to older estimates. Models with heavier cores have slightly steeper and brighter HBs, e.g. Caloi et al.'s (1997) non-linear $M_{V}-[\mathrm{Fe} / \mathrm{H}]$ relation which can be approximately characterized by

$$
M_{V}(Z A H B)=0.26([\mathrm{Fe} / \mathrm{H}]+1.6)+0.49
$$

Caputo et al.'s (1993) and slightly adjusted Caputo (1997) HB models predict a break in slope which occurs near $[\mathrm{Fe} / \mathrm{H}]=-1.3$ if RR Lyrae stars have scaled solar abundance of $\alpha$ elements, and near $[\mathrm{Fe} / \mathrm{H}]=-1.6$ if $\alpha$ elements are enriched by a factor of $2-3$. These models predict a steep slope of 0.3 for metal-rich RR Lyrae stars and a shallow slope of 0.19 for metalpoor stars. The zero point favors the short distance scale with $\mu_{L M C} \approx 18.4$. Caputo (1997) checks her synthetic HB evolutionary models proving that they satisfy the constraints produced by convective pulsating models with a range of masses and He abundance $Y=0.24$. Such models allow one to predict the borders of the RR Lyrae distribution in the $P-M_{V}$ plane. Moreover, Caputo (1997) concludes that adopting the "long distance scale" with a steep slope for all metallicities $\left(M_{V}=0.3[\mathrm{Fe} / \mathrm{H}]+0.94\right)$ results in $\mathrm{RRc}$ stars falling in the hot stable region, i.e. outside the instability strip. On the other hand, the calibrations with a shallow slope are in agreement with observations only if the zero point suggested by Fernley (1994) is adopted instead of a fainter value.

Lee, Demarque \& Zinn (1990) and Caputo et al. (1993) analyzed HB evolutionary tracks showing explicitly how RR Lyrae luminosity depends on evolutionary stage and that blue, less-massive HB stars cross instability strip at significantly higher $L$ than the ZAHB. Therefore the average RR Lyrae luminosity will depend on which part of the HB they come from and so what is the HB morphology. Particular $M_{V}-[\mathrm{Fe} / \mathrm{H}]$ relations will therefore in general depend on the HB morphology of the cluster. If the cluster contains $B$ blue HB stars, $V$ RR Lyrae stars, and $R$ red HB stars, then its morphology index (Lee 1989), $(B-R) /(B+V+R)$, is a number in the interval $(-1,1)$. Morphologies below -0.8 (47 Tuc) and above 0.8 (M92) indicate very red and very blue HBs and, according to Caputo (1997), are likely to complicate the picture. However, even if one restricts oneself to intermediate morphologies, the uncertainties of HB models (at least 0.1 $\mathrm{mag} / \mathrm{dex}$ in slope and $0.2 \mathrm{mag}$ in zero point) prohibit any precise conclusions about the theoretically-preferred $M_{V}-[\mathrm{Fe} / \mathrm{H}]$ relation.

There is also another theoretical path leading to a determination of $M_{V}$. It progresses through the analysis of double mode RR Lyrae stars which is rather complex and is beyond the scope of this paper. Representative results (Alcock et al. 1997, Kovács \& Walker 1998) tend to give a bright $M_{V}$. 


\section{Main or White-Dwarf Sequence Fitting to Globular Clusters}

The main sequence (MS) or white-dwarf (WD) cooling-sequence fitting techniques give the distance to a cluster by matching the cluster sequence of subdwarfs or WDs to a corresponding sequence in $M_{V}$ - dereddened color plane as determined from calibrating stars in the solar neighborhood. The $M_{V}$ of the HB can be determined from the equation:

$$
\begin{aligned}
M_{V}(R R) & =V(R R)-A_{V}(R R)-\left[V(C S)-M_{V}(L C)-A_{V}(C S)+\sum_{i} \frac{d M_{V}}{d \gamma_{i}} \delta \gamma_{i}\right] \\
& \approx V(R R)-\left[V(C S)-M_{V}(L C)+\sum_{i} \frac{d M_{V}}{d \gamma_{i}} \delta \gamma_{i}\right]
\end{aligned}
$$

where $V(R R)$ and $V(C S)$ are the apparent magnitudes of RR Lyrae stars in a cluster and the cluster sequence, respectively, $A_{V}(R R)$ and $A_{V}(C S)$ are the visual extinctions toward them, and $M_{V}(L C)$ is the $M_{V}$ of the local calibrators at a given dereddened color. Finally, $\sum_{i} \frac{d M_{V}}{d \gamma_{i}} \delta \gamma_{i}$ corrects for the fact that for cluster stars it is not always possible to find local calibrators with the same characteristics (parameter $\gamma_{i}$ ) or just for parameter uncertainty of order of $\delta \gamma_{i}$. For MS fitting the most essential parameters are metallicity and color (e.g., $(B-V)$ ), for WD fitting - mass and color. Note that if the cluster sequence stars experience the same extinction as RR Lyrae star, then in equation (18), $A_{V}(R R)$ and $A_{V}(C S)$ cancel out. In practice it is enough if MS or WD sequence stars are drawn from the same region of the cluster as RR Lyrae stars. There are a few crucial criteria that must be met to make the fitting method work properly:

1. accurate distances to the local calibrators must be known. Only when one knows the distances is it possible to construct MS or WD curves in the $M_{V}$ - dereddened color plane. In principle one should also know the reddenings to individual stars, but for the calibrating stars, most of which sit in the Local Bubble, this is not a major concern.

2. if the results of the sequence fitting are sensitive to some parameter, numerous stars in different ranges of this parameter should be available. Metallicity is a key parameter for the MS fitting because the $M_{V}$ of the MS at the given color is a function of metallicity, especially at the high metallicity end. One wants to avoid using theoretical models to shift MS defined at one metallicity to get the $M_{V}$ for another.

3. one must measure the parameters of the cluster on the same scale as applied to the local calibrators, e.g., the metallicity of a cluster should be measured on the same scale as the one used for local subdwarfs. It is necessary to match the appropriate local sequence to the one observed in a cluster. One possible cause of a systematic error in the MS fitting distances is that the metallicities of the local subdwarfs might be on 
a different scale from those of the clusters (determined from giants). Even if the procedure to obtain each type of metallicity is completely uniform, the conclusions depend on the atmospheric models, which are completely different for subdwarfs and giants. Specifically, if the subdwarf metallicities were too low (or the giant metallicities too high) then the cluster distance and the HB luminosities would be overestimated. King et al. (1998) found intriguing evidence of a possible misalignment of this sort. They measured the metallicities of M92 subgiants (unfortunately not subdwarfs, but with higher gravity than giants), and obtained metallicities up to half a dex lower than those of M92 giants. Pinnsoneault (1998, priv. comm.) finds a metallicity of M5 from $B V I$ colors of MS stars. His value is $\sim 0.3$ dex more metal-poor than Gratton et al.'s (1997) giant-based determination.

4. reddening corrections to the color must be known very accurately. Reddening changes the conclusions about the $M_{V}$ of MS or WD stars in two major ways. First, through the extinction $A_{V}$, it directly dims the light of the observed stars shifting the cluster sequence vertically on the color magnitude diagram. However, this effect almost entirely cancels the $A_{V}$ correction when one determines the $M_{V}$ of the HB - both types of stars are extincted by approximately the same amount. And second, uncertainty in color, $\delta(X-Y)$, results in the shift in $M_{V}$ of order of $\delta M_{V} \sim \frac{d M_{V}}{d(X-Y)} \cdot \delta(X-Y)$. This effect is particularly dangerous if the $M_{V}$ - color relation is very steep (e.g., for $(B-V)$ color of MS stars, $\left.\delta M_{V} \sim-5 \cdot \delta(B-V)\right)$.

\subsection{MAIN SEQUENCE FITTING}

We stress at the outset that the MS fitting technique is very sound when applied to nearby open clusters. For a given metallicity there are plenty of local calibrating dwarfs that define well constrained sequences. Because they are numerous there is no problem with choosing a sample with very accurate parallaxes and so small errors in $M_{V}$. On the cluster side - because open clusters are close, the spectroscopy of the MS stars is straight forward. Therefore, the clusters and local calibrators are easily put on the same metallicity scale. Globular clusters pose much more severe problems. Their metallicities are generally very low and, consequently, there are few local subdwarfs that can define metal-weak MS's. The local subsample is collected from a larger region, which translates into larger parallax errors. Therefore some of the distances to individual stars require a substantial Lutz-Kelker type correction (Lutz \& Kelker 1973; Hanson 1979; Smith 1987). Additionally, globular cluster are far away and their MS's are too faint for high-resolution spectroscopy. Therefore the cluster metal- 
licities come from giants and not from subdwarfs. Reddening to globular clusters is usually measured from integrated photometry (e.g., Zinn 1980; Reed, Hesser, \& Shawl 1988), and potentially more accurate Strömgren photometry is generally available only for southern clusters. Moreover, for unknown reasons, Strömgren-photometry-based reddenings are systematically smaller than other determinations. As we mentioned above, reddening uncertainties can dramatically affect the distance modulus.

Reid (1997) uses 15 subdwarfs with Hipparcos parallaxes accurate to at least $12 \%$ to define local subdwarf sequences of known $M_{V}$. He separates the calibrating stars into two metallicity bins: intermediate with $[\mathrm{Fe} / \mathrm{H}] \sim-1.4$ on the Zinn \& West (1984) scale and metal-poor with $[\mathrm{Fe} / \mathrm{H}] \sim-2.1$. That is, Reid (1997) does not apply any theoretical transformations to obtain the $M_{V}$ of MS stars at a given color. He reports that observational sequences are much less sensitive to metallicity than predicted by theoretical isochrones. However, this effect may be spurious because Reid (1997) uses Carney (1994) metallicities that are based on low-resolution spectra and therefore are likely to show more metallicity spread than is really present. He derives new distances to 7 globular clusters: M5, NGC 6752, M13, M15, M92, M30, and M68 obtaining distance moduli of 14.45, 13.17, 14.48, 15.38, $14.93,14.95$, and 15.29 , respectively, with formal uncertainties of $0.1 \mathrm{mag}$. For the last four globulars which are metal poor, these new distance moduli are about 0.3 mags higher than the previous standard values. This leads to intrinsically bright HB's (e.g., $M_{V}=0.44 \pm 0.08$ at $[\mathrm{Fe} / \mathrm{H}]=-1.6$ ) with an extremely steep $M_{V}-[\mathrm{Fe} / \mathrm{H}]$ slope of 0.5 .

Gratton et al. (1997) have a bigger local sample, but still believe that they do not have enough calibrating subdwarfs to make completely model independent fits to globular clusters. Instead, using calibrating subdwarfs, they determine the relation between $(B-V)$ color for unevolved MS stars as a function of metallicity. Then they use this relation to find the location of the MS on $M_{V}$ - color diagram. Gratton et al. (1997) determine distances to 9 globular cluster: 6 coinciding with Reid (1997) [except M15], plus 47 Tuc, NGC 288, and NGC 362. Gratton et al. (1997) distances are generally slightly shorter than the ones by Reid (1997), but they seem to be systematically shorter by 0.2 mag for the three of four metal poor clusters: M30, M68, and M92. Gratton et al. (1997) use the metallicity scale of Carreta \& Gratton (1997) which is more metal-rich than Zinn \& West (1984) scale adopted by Reid. All these modifications lead to,

$$
M_{V}(R R)=(0.18 \pm 0.09)([\mathrm{Fe} / \mathrm{H}]+1.6)+(0.45 \pm 0.04) .
$$

where we report not the result from original paper but rather the corrected relation as reported by Gratton (1998). Equation (19) implies $\mu_{L M C} \approx$ $18.60 \pm 0.07$. 
Studies by Reid (1997) and Gratton et al. (1997) devote a lot of effort to correcting for possible systematic effects like Lutz-Kelker corrections, binary contamination, and errors in reddening determinations. Both studies use very similar input data. Nevertheless, they attribute their corrections to the pre-Hipparcos MS fitting results to two different sources. Gratton et al. (1997) argue that smaller parallaxes to calibrating subdwarfs by themselves account for 0.2 mag larger distances to globular clusters. Reid (1997) sees the same trend (Hipparcos magnitudes smaller than the ground-based measurements), but attributes this change mostly to the lack of sensitivity of the subdwarf luminosity to metallicity for stars with $-1.5 \gtrsim[\mathrm{Fe} / \mathrm{H}] \gtrsim-2.0$

(which is very unlikely from a theoretical point of view).

There is, however, some evidence that those two comprehensive studies are not the ultimate in MS fitting analysis following the Hipparcos breakthrough. Pont et al. (1998) opt for using evolved stars and binaries in their analysis, which dramatically increases the number of calibrating stars. They find $\mu_{\mathrm{M} 92}=14.67$, resulting in $\beta \sim 0.6$ [compare to eq. (19)]. Using only unevolved calibrating stars, Pinnsoneault (1998, priv. comm.) argues for a similar $\beta$ from multicolor analysis of M5 (see also above).

\subsection{WHITE DWARF SEQUENCE FITTING}

The main theoretical difficulty one encounters using WD cooling sequences is the dependence of their magnitude on WD mass. Renzini et al. (1996) list four independent observations that constrain WD masses: luminosities of the red giant branch tip, the HB, the asymptotic giant branch (AGB) termination and the post-AGB stars. All of them seem to indicate that masses of WD's in globular clusters $\left(M_{C W D}\right)$ are independent of the host cluster metallicity (excellent!) and approximately equal to $M_{C W D}=0.53 \pm$ $0.02 M_{\odot}$ (Renzini \& Fusi Pecci 1988). (Such a narrow range of masses has never been either confirmed or falsified observationally, e.g., Cool, Piotto \& King 1996 claim that their data for NGC 6397 are consistent with the wider range $M_{C W D}=0.55 \pm 0.05 M_{\odot}$, although they allow that all the spread may originate from the observational errors.) Wood's (1995) theoretical models suggest that the $M_{V}$ of the WDs scales with the WD mass as

$$
\delta M_{V} \sim 2.4 \delta M_{W D}
$$

Therefore, a serious misestimate of the white dwarf $M_{V}$ 's requires a very severe violation of the WD mass constraints coming from a wide variety of theoretical predictions. According to Renzini \& Fusi Pecci (1988) the $M_{V}$ uncertainty produced by differences of WD mass in different globular clusters can be of order of 0.05 ; if a rather conservative range $M_{C W D}=$ $0.55 \pm 0.05 M_{\odot}$ for the mass of the cluster WDs is adopted, $\delta M_{V}$ can reach 
0.12. Nevertheless, all observers agree that within a given system, the distribution of masses is very narrow. This tendency is confirmed by the local white dwarfs used to calibrate the $M_{V}$ of the WD cooling sequence (Bergeron, Saffer, \& Liebert 1992; Bragaglia, Renzini, \& Bergeron 1995). For the solar neighborhood the mean and dispersion of the WD masses are $\left\langle M_{W D}\right\rangle=0.59 \pm 0.1$, but this does not contradict the Renzini \& Fusi Pecci (1988) claim of a very narrow mass range in globular clusters, where WD populations come from low-mass progenitors only.

There may be also some photometric errors leading to misestimates of $(B-V)$, but they are not likely to shift $M_{V}$ by more than 0.1 mag (in a random direction). Moreover, WDs have practically metal-free atmospheres that come in two varieties: pure H (DA) constituting the great majority and pure He (DB), which makes modeling very straight forward. They are more abundant than subdwarfs and consequently it is much easier to collect a large calibrating sample with accurate trigonometric parallaxes. There is, however, one main observational disadvantage of using WD cooling sequences, namely they are at least 10 mag fainter than MS stars of the same temperature. For the closest globular clusters, WDs have $V \gtrsim 23$ (De Marchi, Paresce, \& Romaniello 1995; Richer et al. 1995, 1997; Cool et al. 1996) and so HST is the best tool to acquire photometric data.

Even though WD sequences have been observed for at least 6 globular clusters (47 Tuc, $\omega$ Cen, NGC 6397, M15, M4, NGC 6752), the distance to only one globular cluster was determined by the WD fitting technique (Renzini et al. 1996 for NGC 6752), one result was mentioned parenthetically (Richer et al. 1997 for M4) and there exist persistent rumors about one not yet published (Renzini 1998 for 47 Tuc). Renzini et al. (1996) match the WD sequences (when DA is matched, DB falls in the right place) of NGC 6752 with the local WD sequence and find $\mu_{N 6752}=13.05$. When combined with the reddening of the cluster and apparent magnitude of the HB from Gratton et al. (1997) as originally observed by Penny \& Dickens (1986), this yields $M_{V}(R R)=0.59$ at $[\mathrm{Fe} / \mathrm{H}]=-1.4$. Renzini (1998) has also recently reported a distance modulus to 47 Tuc that is about 0.5 mag shorter than the distance obtained through MS fitting and would imply $M_{V}(R R)=0.95$ at $[\mathrm{Fe} / \mathrm{H}]=-0.7$. Richer et al. (1997) mention that if they use Renzini \& Fusi Pecci (1988) cluster white dwarf mass estimate of $M_{W D}=0.53 \pm 0.02 M_{\odot}$, their (dereddened) distance modulus to M4 will be 11.09. Adjusting the HB $M_{V}$ of Rees (1996) to match the Richer et al. (1997) distance modulus to M4, one obtains $M_{V}=0.75$ at $[\mathrm{Fe} / \mathrm{H}]=-1.3$. Due to the very small number of analyzed clusters, the WD cooling sequence technique must be interpreted cautiosly, but nevertheless seems to give some support to the short RR Lyrae distance scale. For the 3 clusters (and fixing the slope of $M_{V}-[\mathrm{Fe} / \mathrm{H}]$ relation at 0.2 ), we find 
$M_{V}(R R)=0.67 \pm 0.13$ at $[\mathrm{Fe} / \mathrm{H}]=-1.6$.

\section{Summary}

TABLE 1. Comparison between different methods to determine RR Lyrae absolute magnitudes

\begin{tabular}{|c|c|c|c|c|}
\hline Method & $\begin{array}{c}\text { Absolute } \\
\text { magnitude } \\
\text { at }[\mathrm{Fe} / \mathrm{H}]=-1.6\end{array}$ & Grade & Main problems & $\begin{array}{l}\text { Potential } \\
\text { future } \\
\text { usefulness }\end{array}$ \\
\hline Statistical parallax & $0.77 \pm 0.13$ & $\mathrm{~A}$ & - & $\mathrm{A}-$ \\
\hline Trigonometric parallax & $0.71 \pm 0.15$ & $A-$ & $\begin{array}{l}\text { done for non-RR Lyrae stars } \\
\text { small number statistics }\end{array}$ & $\mathrm{A}+$ \\
\hline Cluster kinematics & $0.67 \pm 0.10$ & $\mathrm{~B}+$ & $\begin{array}{l}\text { modeling of rotation } \\
\text { uncertain density profile } \\
\text { proper motions }\end{array}$ & $A-$ \\
\hline Baade-Wesselink & $0.45-0.70$ & $\mathrm{~B}$ & $\begin{array}{l}\text { temperature scale } \\
p \text { factor, bolometric corrections }\end{array}$ & $\mathrm{B}$ \\
\hline Theoretical models & $0.45-0.65$ & $\mathrm{~B}-$ & input physics & $\mathrm{A}$ \\
\hline Main sequence fitting & $0.45 \pm 0.04$ & $\mathrm{C}$ & $\begin{array}{l}\text { metallicity scale } \\
\text { reddening uncertainties } \\
\text { small number of calibrators }\end{array}$ & $\mathrm{B}$ \\
\hline White dwarf fitting & $0.67 \pm 0.13$ & $\mathrm{C}$ & $\begin{array}{l}\text { WD masses from theory } \\
\text { reddening uncertainties }\end{array}$ & $\mathrm{A}$ \\
\hline
\end{tabular}

The most crucial outcome of the Hipparcos mission is the realization that astrometry is at the moment the only fully reliable way to measure distances in the local Universe. However, instead of removing a long-standing discrepancy among existing distance scales, Hipparcos results led to numerous analyses which have strengthened old conflicts and created new ones. Table 1 summarizes the results reported in this review. Note that the errors quoted are statistical only. We have ranked and graded (A - very good; B good; $\mathrm{C}$ - acceptable) the methods by our judgement of their susceptibility to systematic effects and also included our grade of their potential reliability with forseeable improvement in the data. Of the methods listed only 3 (statistical parallax, trigonometric parallax and MS fitting) have been affected by Hipparcos.

This work was supported in part by grant AST 97-27520 from the NSF. 


\section{References}

Ajhar, E.A., Grillmair, C.J., Lauer, T.R., Baum, W.A., Fabar, S.M., Holtzman, J.A., Lynds, R.C., \& O’Neill, Jr., E.J. (1996), Astronomical Journal, 111, 1110

Alcock, C. et al. (1997), Astrophysical Journal, 482, 89

Beers, T.C., \& Sommer-Larsen, J. (1995), Astrophysical Journal Supplement, 96, 175

Bergeron, P., Saffer, R., \& Liebert, J. (1992), Astrophysical Journal, 394, 228

Bono, G., Caputo, F., Castellani, V., \& Marconi, M. (1995), Astrophysical Journal, 448, L115

Bragaglia, A., Ranzini, A., \& Bergeron, P. (1995), Astrophysical Journal, 443, 735

Burstein, D., \& Heiles, C. (1982), Astronomical Journal, 87, 1165

Cacciari, C., Clementini, G., \& Fernley, J. (1992), Astrophysical Journal, 396, 219

Caloi, V., D'Antona, F., Mazzitelli, I. (1997), Astronomy \& Astrophysics, 320, 823

Caputo, F. (1997), MNRAS, 284, 994

Caputo, F., De Rinaldis, A., Manteiga, M., Pulone, L., \& Quarta, M.L. (1993), Astronomy E Astrophysics, 276, 41

Caretta, E., \& Gratton, R.G. (1997), Astronomy \& Astrophysics Supplement, 121, 95

Carney, B.W., Latham, D.W., Laird, J.B. (1994), Astronomical Journal, 107, 2240

Catelan, M. (1998), Astrophysical Journal, 495, L81

Clube, S.V.M., \& Dawe, J.A. (1980), MNRAS, 190, 575

Cool, A.M., Piotto, G., \& King, I.R. (1996), Astrophysical Journal, 468, 655

Cudworth, K.M. (1979), Astronomical Journal, 84, 1313

Cudworth, K.M., \& Monet, D.G. (1979), Astronomical Journal, 84, 774

D'Antona, F., Caloi, V., \& Mazzitelli, I. (1997), Astrophysical Journal, 477, 519

De Marchi, G., Paresce, F., Romaniello, M. (1995), Astrophysical Journal, 440, 216

Duquennoy, A., \& Mayor, M. (1991), Astronomy \& Astrophysics, 248, 485

Feast, M.W. (1997), MNRAS, 284, 761

Fernley, J. (1994), Astronomy \&6 Astrophysics, 284, L16

Fernley, J., Barnes, T.G., Skillen, I., Hawley, S.L., Hanley, C.J., Evans, D.W., Solano, E., \& Garrido, R. (1998a), Astronomy \& Astrophysics, 330, 515

Fernley, J., Carney, B.W., Skillen, I., Cacciari, C., \& Janes, K. (1998b), MNRAS, 293, L61

Fernley, J., Lynas-Gray, A.E., Skillen, I., Jameson, R.F., Kilkenny, D., Marang, F., \& Longmore, A.J. (1989), MNRAS, 236, 447

Fusi Pecci, F. et al. (1996), Astronomical Journal, 112, 1461

Getting, I.P. (1935), MNRAS, 95, 141

Gould, A., \& Popowski, P. (1998), Astrophysical Journal, submitted (astro-ph/9805176)

Gratton, R.G. (1998), MNRAS, submitted (astro-ph/9710271)

Gratton, R.G., Fusi Pecci, F., Carreta, E., Clementini, G., Corsi, C.E., Lattanzi, M. (1997), Astrophysical Journal, 491, 749

Gunn, J.E., \& Griffin, R.F. (1979), Astronomical Journal, 84, 752

Hanson, R.B. (1979), MNRAS, 186, 875

Hawley, S.L., Jeffreys, W.H., Barnes, T.G. III, \& Wan, L. (1986), Astrophysical Journal, 302, 626

Hazen, M.L. \& Nemec, J.M. (1992), Astronomical Journal, 104, 111

Hut, P., et al. (1992), PASP, 104, 981

Jones, R.V., Carney, B.W., Storm, J., \& Latham, D.W. (1992), Astrophysical Journal, 386, 646

Karp, A. (1975), Astrophysical Journal, 201, 641

King, J.R., Stephens, A., Boesgaard, A.M., \& Deliyannis, C.P. (1998), Astronomical Journal, 115, 666

Klemola, A.R., Hanson, R.B., Jones, B.F.(1993), Lick Northern Proper Motion Program: NPM1 Catalog, Astronomical Data Center catalog No. A1199

Kovács, G., \& Jurcsik, J. (1996), Astrophysical Journal, 466, L17

Kovács, G., \& Walker, A. (1998), submitted to Astrophysical Journal (available at 
http://www .konkoly.hu/staff/kovacs.html)

Kurucz, R.L., (1992), in Barbuy, B., Renzini, A., eds, The Stellar Population of Galaxies, (Kluwer:Dordrecht), p. 225

Layden, A.C. (1994), Astronomical Journal, 108, 1016

Layden, A.C., Hanson, R.B., Hawley, S.L., Klemola, A.R., \& Hanley, C.J. (1996), Astronomical Journal, 112, 2110

Lee, Y.-W. (1989), PhD thesis, Yale University

Lee, Y.-W., Demarque, P., \& Zinn, R. (1990), Astrophysical Journal, 350, 155

Liu, T., \& Janes, K.A. (1990), Astrophysical Journal, 354, 273

Lupton, R.H., Gunn, J.E., \& Griffin, R.F. (1987), Astronomical Journal, 93, 1114

Lutz, T.E. \& Kelker, D.H. (1973), PASP, 85, 573

Mazzitelli, I., D'Antona, F., \& Caloi, V. (1995), Astronomy \& Astrophysics, 302, 382

McNamara, D.H. (1997), PASP, 109, 857

Mitchie, R.W. (1963), MNRAS, 125, 127

Penny, A.J., \& Dickens, R.J. (1986), MNRAS, 220, 845

Peterson, R.C., \& Cudworth, K.M. (1994), Astrophysical Journal, 420, 612

Pont, F., Mayor, M., Turon, C., \& VandenBerg, D.A. (1998), Astronomy \& Astrophysics, 329, 87

Popowski, P., \& Gould, A. (1998a), Astrophysical Journal, 506, 000 (astro-ph/9703140)

Popowski, P., \& Gould, A. (1998b), Astrophysical Journal, 506, 000 (astro-ph/9802168)

Preston, G.W., \& Paczyński, B. (1964), Astrophysical Journal, 140, 181

Pryor, C., McClure, R.D., Fletcher, J.M., \& Hesser, J.E. (1989), Dynamics of Dense Stellar Systems, D. Merritt, ed., 175 (Cambridge: Cambridge Univ. Press)

Reed, B.C., Hesser, J.E., \& Shawl, S.J. (1988), PASP, 100, 545

Rees, R.F. (1996), Formation of the Galactic Halo Inside and Out, H. Morrison and A. Sarajedian, eds., 289 (San Francisco: ASP)

Reid, I.N. (1997), Astronomical Journal, 114, 161

Reid, N., Freedman, W. (1994), MNRAS, 267, 821

Renzini, A., Bragaglia, A., Ferraro, F.R., Gilmozzi, R., Ortolani, S., Holberg, J.B., Liebert, J., Wesemael, F., \& Bohlin, R.C. (1996), Astrophysical Journal, 465, L23

Renzini, A., \& Fusi Pesci, F. (1988), ARA\&A A, 26, 199

Richer, H.B. et al. (1995), Astrophysical Journal, 451, L17

Richer, H.B. et al. (1997), Astrophysical Journal, 484, 741

Salaris, M., Degl'Innocenti, S., Weiss, A. (1997), Astrophysical Journal, 479, 665

Sandage, A. (1970), Astrophysical Journal, 162, 841

Sandage, A. (1981), Astrophysical Journal, 248, 161

Sandage, A. (1982), Astrophysical Journal, 252, 553

Sandage, A. (1993), Astronomical Journal, 106, 703

Schlegel, D.J., Finkbeiner, D.P., \& Davis, M. (1998), Astrophysical Journal, 500, 525

Skillen, I., Fernley, J., Stobie, R.S., \& Jameson, R.F. (1993), MNRAS, 265, 301

Smith, H. (1987), Astronomy \& Astrophysics, 188, 233

Smith, H.A. (1995), RR Lyrae stars (Cambridge University Press: Cambridge)

Storm, J., Carney, B.W., Latham, D.W. (1994), Astronomy \& Astrophysics, 290, 443

Tsujimoto, T., Miyamoto, M., \& Yoshii, Y. (1998), Astrophysical Journal, 492, L79

VandenBerg, D.A., Swenson, F.J., Rogers, F.J., Iglesias, C.A., \& Alexander, D.R. (1998), in preparation

Walker, A.R. (1992), Astrophysical Journal, 390, L81

Wood, M.A. (1995), in White Dwarfs, ed. D. Koester \& K. Werner (Berlin: Springer), 41 Zinn, R. (1980), Astrophysical Journal Supplement, 42, 19

Zinn, R., \& West, M.J. (1984), Astrophysical Journal Supplement, 55, 45 